\title{
On the possible production and detection of coherent low-energy neutrino beams
}

\author{
Jeff W. Eerkens
}

Received: 31 December 2012/ Accepted: 11 January 2013/Published online: 5 February 2013

(C) The Author(s) 2013. This article is published with open access at Springerlink.com

\begin{abstract}
Seminal experiments are discussed to prove or disprove the possibility of producing and detecting collimated coherent beams of low-energy neutrinos with the assistance of lasers. Expressions are given for the relative probability that a laser-beam-aligned co- or counterpropagating neutrino-antineutrino pair is emitted instead of a laser photon, during stimulated de-excitations of lasable excited states inside a lasing medium. For a neodymium laser this probability is approximately $10^{-7}$. To detect coherent beams of epithermal neutrinos and antineutrinos emitted from a pulsed high-power laser, it is anticipated that resonant non-absorptive stimulated de-excitations of lasing levels by neutrinos can be exploited to register their fly-through in a second near-threshold laser.
\end{abstract}

\section{Introduction and history}

The concept of producing and detecting collimated coherent beams of low-energy neutrinos with the assistance of lasers was first proposed [1] by the author in 1967 and incompletely explored in interrupted lab experiments in 1971. The main difference between the present re-investigation and the one in 1967 is that validity of the old neutrino theory of photons (NTP), once investigated by Max Born, Louis DeBroglie, R. Kronig, and other physics luminaries, [2-9] is not invoked as a co-requirement for the validity of generating possible coherent neutrino beams. As illustrated in Fig. 1, it is

J. W. Eerkens

Prodev Consultants, PO Box 8566, Woodland, CA 95776, USA

J. W. Eerkens $(\bowtie)$

Nuclear Science and Engineering Institute,

University of Missouri, Columbia, MO 65211, USA

e-mail: eerkensj@missouri.edu conjectured that emissions of co- or counter-propagating recoiling neutrino-antineutrino pairs can be a substitute for photon-emitting atomic de-excitations, but with many orders-of-magnitude lower probability than the photon emissions. To detect such neutrinos, stimulated resonant de-excitation instead of absorption is proposed as the basic mechanism for detection. Straight-through-the earth neutrino-carried communications between ships, aircraft, satellites, and land-based stations would become feasible if one can generate and modulate coherent neutrino beams, and can observe (demodulate) their presence. Detection and demodulation appears possible by utilizing fly-through neutrino-stimulated de-excitations (no need to catch them) of lasing atoms or molecules in near-threshold lasers. Clearly, besides advancing physics, a large number of new applications can be envisioned for coherent neutrino beams, some of which were reviewed in a patent issued in 1980 [10].

The neutrino has never been fully exploited primarily because it is very difficult to detect and observe. It may be likened to the photon in that it appears to move at the speed of light with no mass. Its spin is $s=1 / 2 \hbar$, whereas a photon has spin $s=0, n \hbar$, where $n$ is an integer. It appears not to react with the electromagnetic field due to the fact it is not absorbed by materials. In an ordinary photon absorption process, an integral spin change of $\Delta s= \pm n \hbar$ is required to conserve spin. Since, a single neutrino has only spin $s=1 / 2 \hbar$, it is not absorbable. It allows neutrinos to pass straight through the earth un-impeded. However, this restriction does not prevent a resonant neutrino to stimulate the de-excitation of an excited ion, atom, or molecule in a fly-by event without being absorbed. In short, while photons with integral spin $s=0,1$ can be absorbed or emitted by matter in molecular interactions that demand $\pm \Delta s=0$, $1, \ldots$, neutrinos with $s=1 / 2$ can still stimulate de-excitations without being absorbed. 


$$
\begin{aligned}
& \hat{A}_{v}=A_{o}\left[-\hat{e}_{x} \sin \left(k_{z} z+\omega t\right)+\hat{e}_{y} \cos \left(k_{z} z+\omega t\right)\right] \\
& \hat{A}_{\underline{v}}=A_{o}\left[\hat{e}_{x} \sin \left(k_{z} z-\omega t\right)+\hat{e}_{y} \cos \left(k_{z} z-\omega t\right)\right]
\end{aligned}
$$

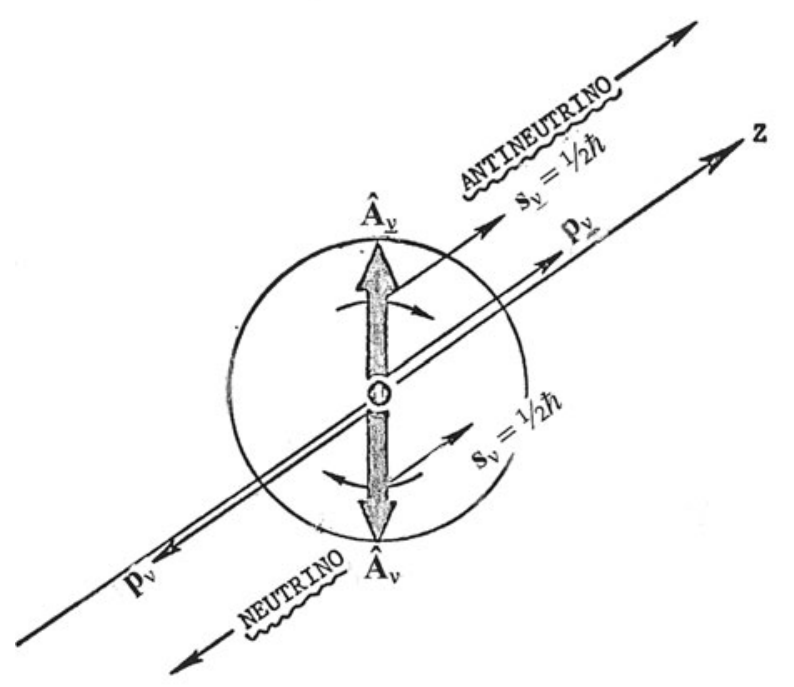

(a) Neutrino-Antineutrino Pair leaving Atom in Opposite Directions after Creation

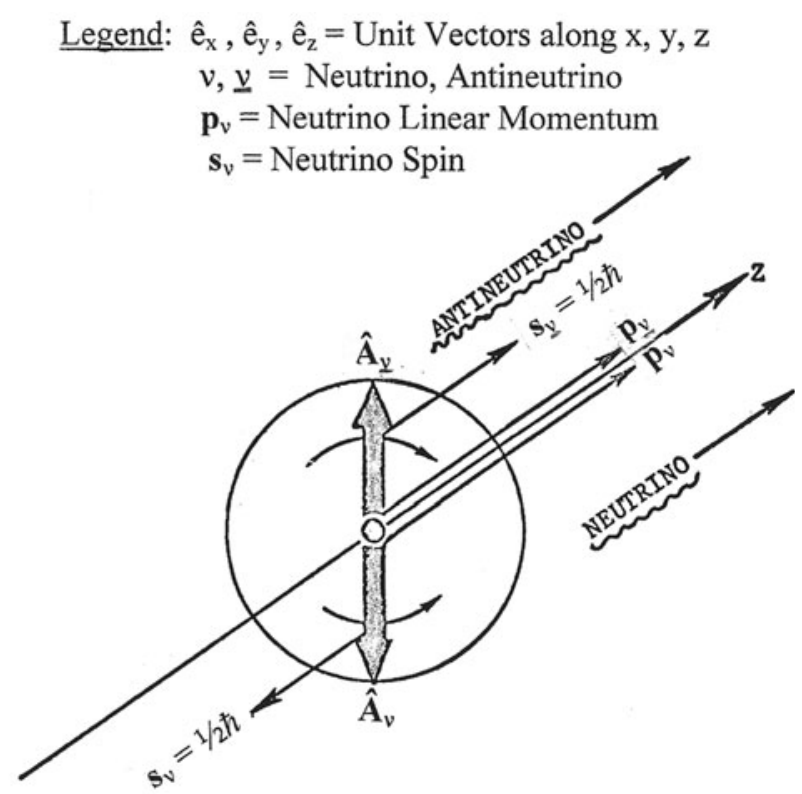

(b) Neutrino-Antineutrino Pair leaving Atom in Same Direction after Creation [The Neutrino Theory of Photons (NTP) postulates such a Co-traveling Neutrino/Antineutrino Pair constitutes a Photon]

Fig. 1 Classical depiction of neutrino-antineutrino-pair emission

Neutrino research to date has focused mainly on neutrinos with energies in the $\mathrm{MeV}$ region, produced in beta decay and other nuclear processes in reactors, accelerators, the sun, and various stellar bodies. Three neutrino "flavors" have been observed, namely those produced in association with beta decay (e-neutrinos), those with muon decay ( $\mu$-neutrinos) and those with tau-particle decay ( $\tau$-neutrinos). The energy spectrum of neutrinos emitted in beta decay is not discrete and varies continuously between 0 and $8 \mathrm{MeV}$. That is, low-energy eV neutrinos do exist and are emitted in great numbers but are virtually unexplored. Only $\mathrm{MeV}$ neutrinos have been investigated so far because their presence can be (and are) observed by inverse beta-decay absorptions in nuclei, in spite of very low crosssections that demand enormously large scintillator detection volumes. In contrast, if verified, the generation and detection of our low-energy e-(anti)neutrino fluxes requires only a high-power pulsed source laser (SL) for neutrino beam generation, and a low-power $\mathrm{CW}$ laser for detection (DL) as shown in Fig. 2.

Presently it is believed that electron-neutrinos can morph into muon-neutrinos and vice versa, which presumes that (some) neutrinos must have mass. Propagation of electromagnetic quanta through space at the speed of light requires them to be mass-less, however, and the simultaneous arrival (after 170,000 years of travel!) of photons and neutrinos from a supernova event observed on 23 February 1987, proves at least some neutrinos must be mass-less. Thus, we assert that mass-less electromagnetic quanta can be propagated either by photons with spin $s=0,1, \ldots$ (in units of $\hbar$ ) or by neutrinos with $s=1 / 2$. We further adopt the experiment-compatible picture that lowenergy e-neutrinos are mass-less, but that $\mu$ - and $\tau$-neutrinos may have some mass. Mass-less e-neutrinos could morph into $\mu$-neutrinos with mass $m_{\mu}$ only if they are energetic enough (perhaps at supra-keV energies). Knowing that mass-less gammas with energies above $m_{\gamma}=1.022 \mathrm{MeV}$ can morph into massive $\mathrm{e}^{-} \mathrm{e}^{+}$pairs, one might likewise expect that mass-less e-neutrinos could transform into mass-carrying $\mu$-neutrinos with mass $m_{\mu}$ at high energies. If the rest-mass $m_{\mu}$ were known, one can calculate a lower limit for e-neutrino energies below which such morphing into a $\mu$-neutrino is impossible. In what follows we shall only consider sub-keV e-(anti)neutrinos that are mass-less and lack the energy to materialize ("sterile" neutrinos?). We shall omit pre-scripts "e-", since only mass-less electron (anti)neutrinos are under consideration from here on.

In producing and detecting neutrinos, we utilize the process of stimulated emission (SE). Einstein proposed this interaction mechanism in 1917 to supplement then-existing emission-absorption theories for photons. The SE process was originally thought to have only minor effects and was not more thoroughly investigated till the $1950 \mathrm{~s}$, which lead to the birth of the laser in 1960. In lasers, photon undulation frequencies $\omega_{\gamma}=\omega_{\mathrm{L}}$ are resonant with the $n \leftrightarrow k$ 


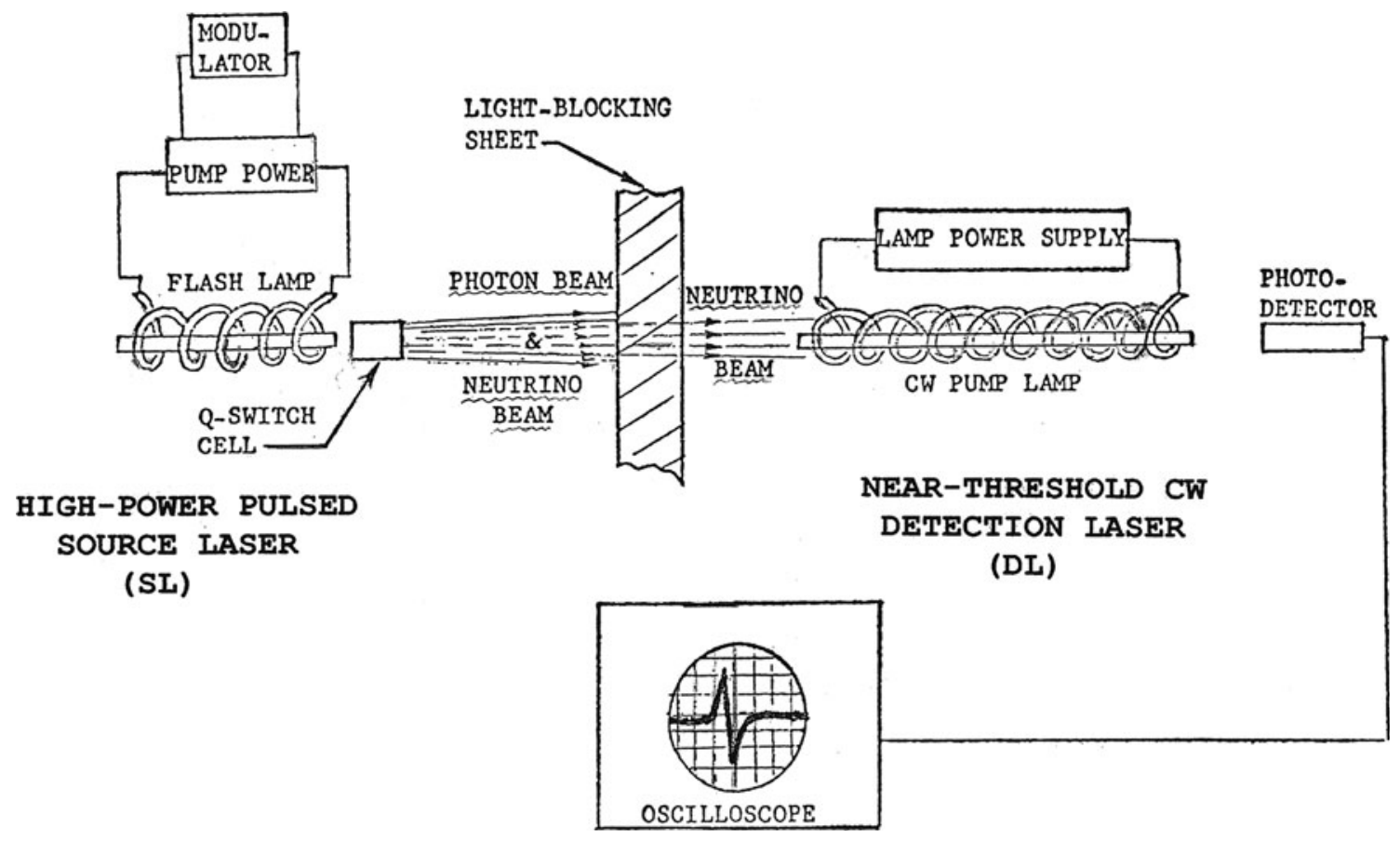

Fig. 2 Experimental coherent neutrino generation and detection system

transition frequency $\omega_{k n}=E_{k n} / \hbar$ of photon-emitting excited ("pumped") atoms or molecules, i.e. $\omega_{\gamma}=\omega_{\mathrm{L}}=\omega_{k n}$. Besides enabling lasers, the SE mechanism was also successfully used in 1958 by Mossbauer to induce gammastimulated emissions from excited nuclei. Laser photons generate secondary laser photons via SE of excited atoms, but are not absorbed in that process (spin exchange is not involved). Inside an optical resonator this promotes photon multiplication and the build-up of a coherent monochromatic laser beam. We now postulate that in a laser, (anti)neutrinos with resonant undulation frequencies $\omega_{v}=\omega_{\underline{v}}=\omega_{k n}=\omega_{\mathrm{L}}$ can also induce photon-emitting $n \leftrightarrow k$ transitions via absorption-less SE that does not involve spin exchange. This opens up the possibility of detecting resonant coherent (anti)neutrino beams discussed further below.

The SE process in lasers might not only allow the direct detection of neutrino "fly-throughs" without their absorption, but it should also generate low-energy neutrino-pair emissions now and then, in place of a laser photon emission. Such emissions produce two co- or counter-propagating neutrinos with equal or opposite momenta and spins, moving in the same or opposite direction (Fig. 1) under the full obedience of all conservation laws for energy, momentum, and spin. This second-order process is similar to a Raman interaction in which a new outgoing photon and exciton are exchanged for one incoming photon. It also resembles in some ways the decay of mesons discussed in a paper by Sternglass [12] titled "Electron-Positron Model of Charged Meson and Pion Resonances". Although the present model with quarks has replaced Sternglass' more physically anthropomorphic theory of 1965 , the agreement of Sternglass' models with measured meson lifetimes and masses gives it a considerable credibility. Similar to Sternglass' concept, who visualizes mass-possessing electrons and positrons as "curled-up" packets of electric matter and anti-matter bundled up in mesons, one might view neutrinos and antineutrinos as "un-furled" mass-less electromagnetic energy quanta that are occasionally released by excitons as an alternative to the emission of a single photon. In short, analogous to some meson decays, the occasional exciton conversion into a neutrino-antineutrino pair seems reasonable. We label the latter " $v \underline{v}$ pairs" with the tacit assumption they recoil in the same or opposite directions.

An earlier version of the present concept was described in a research proposal [1] to ARPA in 1967 and was also disclosed in a patent issued in 1980 [10]. In the earlier version, it was assumed that for neutrino pair production it was necessary to employ a laser medium which had an intermediate energy level half-way between the upper and lower lasing levels. It is now believed this is unnecessary. The earlier proposal was neither funded nor seriously considered because at the time no new government $R \& D$ projects were accepted unless directly related to the Vietnam-War effort. In addition, while no official answer was received from ARPA in 1967, it is believed that peer reviewers (if any) of the proposal, might have been 
skeptical about the validity of its underlying physics which referred to the "Neutrino Theory of Photons" (NTP) to support the possibility that low-energy neutrino pairs might be generated with lasers. In the 1930s, the NTP concept was seriously considered by prominent physicists such as Louis DeBroglie, Max Born, and R. deL. Kronig [2-5], and re-considered in the mid-1960s by Perkins [6] and by Ferretti and Venturi [7]. In this model, it is assumed that photons are composed of co-traveling neutrino-antineutrino pairs (Fig. 1b). However, two respected nuclear physicists, Pryce [8] in a 1937 paper and Berezinski [9] in a 1965 paper rejected the NTP model, showing it did not remain invariant under reference-frame rotations as demanded by basic physics. Reservations of reviewers (if any) of the 1967 proposal were most likely based on Pryce's and Berezinski's objections to the NTP. Although Perkins [11] later rebutted these objections, the concept of generating and detecting epithermal neutrino beams as proposed here does not depend on the NTP.

In 1971, the author decided to execute his proposed experiment without government funds. On a small private budget, two neodymium lasers were rented from the Korad Corporation and tests were carried out for 2 days at Korad's facility in Santa Monica, California. One of the neodymium lasers was a powerful pulsed unit and the other a near-threshold low-power CW (continuous wave) device, arranged as illustrated in Fig. 2 and discussed in the 1967 ARPA proposal document [1]. The Korad tests did not unequivocally prove or reject the conjectured possibility of producing and detecting low-energy neutrinos. When the pulsed laser was fired, a signal was seen at the detector of the CW threshold laser in spite of blockage of the pulsed laser output by a brick to prevent laser photons from entering the $\mathrm{CW}$ unit, while permitting the assumed neutrinos to pass through. However, it is believed these signals were produced by interference via the electric net between the Pockels-Cell trigger of the pulsed laser and the $\mathrm{CW}$ threshold-laser output detector. It was planned to repeat the Korad experiments with avoidance of possible interference between monitoring sensors of the two lasers using isolated batteries. But this plan was never executed due to lack of funds and the closing of Korad's facility a few months later.

As mentioned, the proposed production and detection of low-energy neutrinos does not rely on the old NTP. The co- or counter-propagating $\mathrm{v} v$-pairs leave an atomic or molecular de-excitation event in a laser in directions along the laser beam, and can be considered as two aligned recoiling particles. They do not need to be viewed as half-components of photons. Such less frequent $v \underline{v}$-pair emissions involve three-quantum transitions and are comparable to three-quantum photonic Raman conversions, whose probability is also much smaller than that of a single photon absorption or emission event, but not impossible. Perkins' revival of the NTP concept in 1965 was partly based on a two-fold 2-component neutrino model. However, that model is not explicitly needed in the present concept for assessing the possibility of generating and detecting low-energy neutrinos utilizing lasers. There have been debates in the literature whether the neutrino has two or four distinguishable manifestations, namely: (1) a neutrino with right-handed helicity; (2) a neutrino with lefthanded helicity; (3) an antineutrino with right-handed helicity; (4) an antineutrino with left-handed helicity. If low-energy neutrinos are mass-less as we believe, then like the photon, the only reality is a distinction between lefthanded and right-handed neutrinos. For both the photon and e-neutrino the anti-particle is undistinguishable from the particle and behaves exactly the same, except for helicity. Experimentally, it has been proven that antineutrinos produced in nuclear-reactors due to beta decay have right-handed helicities, while neutrinos emitted by the sun, supernova, and accelerators, spiral left-handedly as they propagate through space.

Although the experimenters were unaware of it, proof that low-energy neutrinos can directly stimulate de-excitations of excited states in lasers without being absorbed, may already have been demonstrated experimentally. Experiments carried out by Compton [13] in 1964 at General Atomics produced unexpected unexplainable results which can be interpreted as due to neutrino-stimulated de-excitations. Compton originally attempted to induce lasing action in neodymium and ruby laser rods using scintillator-emitted pump light activated in turn by reactor gammas. Instead, he found that functioning optically pumped lasers ceased to lase during a reactor pulse when placed close to the core of a pulsed TRIGA research reactor, even if heavily shielded from gamma and neutron irradiations. Compton [13] conducted exhaustive tests to determine if color-center formation and/or other known reactor-radiation effects might have been responsible. All plausible causes were eliminated as being too weak to cause cessation of laser action in the shielded lasers during a reactor pulse. Also, the optically pumped lasers resumed operations again after the reactor pulse had subsided. To date this effect has remained unexplained. It is the author's belief, however, that the effect was due to neutrino-stimulated de-excitation and disturbances of lasing inversions in the neodymium and ruby laser rods. While low-energy sub-keV neutrinos created in nuclear fission are distributed over a wide range of energies (i.e. frequencies), and most were not coincident with the lasing transition frequency, the number of omni-directionally emitted neutrinos in a 4,000 MW millisecond reactor pulse is enormous. A high flux and a sufficiently broadened resonant width for neutrino-stimulated emissions could easily have produced 
excessive de-excitations of upper lasing levels, causing momentary cessations of lasing. At its pulse peak of $4 \mathrm{GW}$, the TRIGA releases approximately $1.5 \times 10^{21}$ neutrinos per second of which perhaps $10^{19}$ neutrinos per second passed through Compton's lasers. The $100 \mathrm{~mW}$ neodymium lasers at $1.06 \mu \mathrm{m}$ of the $1960 \mathrm{~s}$ were pumped with about $5 \times 10^{17}$ excitations per second, so that stimulated de-excitations by a resonant fraction $(\sim 1 \%)$ of the neutrino flux could easily interrupt lasing action.

To support our conjectures, three fundamental phenomena must be experimentally (re)verified: (a) Can neutrinos stimulate the de-excitation of excited states in lasable media; (b) Can stimulated emissions by photons in a laser generate a weak overlapping beam of coherent neutrino/ antineutrino pairs in addition to generating a photonic laser beam; and (c) Is the relation between energy $\in$ and frequency $\omega$ for neutrinos $\epsilon=\hbar \omega$, or is it $\epsilon=1 / 2 \hbar \omega$, or $\mathrm{g} \hbar \omega$ (see below)?

\section{Calculated emission and detection probabilities}

We now summarize theoretical expressions for low-energy neutrino interaction rates that involve (1) spontaneous $v \underline{v}$ pair emissions, (2) photon-stimulated $\underline{v} \underline{v}$ pair emissions, and (3) $v$ - or $\underline{v}$-stimulated de-excitations. Some of the calculations were done more than 40 years ago [1], but little has changed in the recipes used for calculating quantummechanical transition rates. Some errors in the older material were corrected and revised. The following basic assumptions are made about the neutrinos considered here: (a) Lowenergy neutrinos travel at the speed of light and are mass-less; (b) There are only left- and right-handed (anti)neutrinos, most neutrinos from the sun propagate with left-handed spins along their travel path, while anti-neutrinos emitted primarily by nuclear reactors propagate righthandedly. Both neutrinos and anti-neutrinos carry spin $s=1 / 2 \hbar$ ( $\hbar=h / 2 \pi$, where $h$ is Planck's constant); (c) For neutrino detection, the process of stimulated catalytic deexcitation (with photon emission) of atomic or molecular excited states is effective; (d) Neutrino-antineutrino pairs ( $v \underline{v}$ pairs) can be generated in stimulated emissions (SE) as an alternative second-order effect to single photon emissions.

While it has been proven that neutrinos have spin $s=1 / 2 \hbar$, it is not known whether they are propelled with energy packages of $\epsilon_{v}=1 / 2 \hbar \omega_{v}, \epsilon_{v}=\hbar \omega_{v}$, or with $\epsilon_{v}=\mathrm{g} \hbar \omega_{v}$ at undulation frequencies $\omega_{v}\left(=2 \pi v_{v}\right) \mathrm{rad} / \mathrm{s}$. Since, mass-less circularly polarized photons with spin $s=1$ (in units of $\hbar$ ) do exchange energy quanta of $\epsilon_{\gamma}=\hbar \omega_{\gamma}$ and the same holds for photons with $s=0$, one can argue that the energy quanta of spinning (anti)neutrinos with $s=1 / 2$ should also be $\epsilon_{v}=\hbar \omega_{v}$. Furthermore, electrons with spin $s=1 / 2$ undulate at $\omega=\epsilon / \hbar$ as well, though they are not mass-less like low-energy neutrinos. Unfortunately, neutrinos cannot exchange energy packages with atoms so one cannot verify the relation between $\epsilon_{v}$ and $\omega_{v}$ by the photo-electric effect. If the NTP [2-9] was correct, one might be persuaded to assume $\epsilon_{v}=1 / 2 \hbar \omega_{v}$. But even if the NTP is flawed, it does not preclude that $\epsilon_{v}=1 / 2 \hbar \omega_{v}$. Thus, subject to experimental verification, the neutrino's undulation frequency $\omega_{v}$ is assumed to be related to neutrino energy by either $\epsilon_{v}=\hbar \omega_{v}$ or $\epsilon_{v}=1 / 2 \hbar \omega_{v}$. Though unlikely, one might even find that $\epsilon_{v}=\mathrm{g} \omega_{v}$, where $\mathrm{g}$ is a new constant.

\subsection{Spontaneous emission of a neutrino pair}

Assuming a semi-classical model for atomic/molecular transitions, it can be deduced [1] that the spontaneous emission probability of a $v \underline{v}$-pair emission in a transition from upper excited state $\mathrm{k}$ to a lower excited state $\mathrm{n}$ is given by:

$$
\begin{aligned}
{ }^{\text {spon }} \mathrm{k}_{\mathrm{kn}}^{(v \underline{v})} & =\left[\left(2^{7} \pi^{2} / 3\right)\right]\left[\left|\mu_{\mathrm{kn}}\right| /(\hbar c)\right]^{4}\left[E_{\mathrm{kn}}^{5} /\left(\hbar^{5} c^{4}\right)\right] \\
& =\left[\left(2^{7} \pi^{2} / 3\right)\right]\left[\left\{e^{2} /(\hbar c)\right\}^{2}\right]\left[E_{\mathrm{kn}}^{5} /\left(\hbar^{5} c^{4}\right)\right]\left\langle y_{\mathrm{kn}}\right\rangle^{4} \\
& =2.236\left\langle y_{\mathrm{kn}}\right\rangle^{4} E_{\mathrm{kn}}^{5}, s^{-1}
\end{aligned}
$$

Here $E_{\mathrm{kn}}=\mathrm{E}_{\mathrm{k}}-\mathrm{E}_{\mathrm{n}}$ is in eV and $\left\langle y_{\mathrm{kn}}\right\rangle$ is in units of $\AA$ in the last member of (1). The parameter $\left|\mu_{k n}\right|$ is the usual dipole matrix element for an atomic or molecular $\mathrm{k} \rightarrow \mathrm{n}$ transition, which can be equated to $\mathrm{e}\left\langle y_{\mathrm{kn}}\right\rangle$. That is, in the second part of (1) and in all that follows, we prefer to use the displacement matrix element:

$\left\langle y_{\mathrm{kn}}\right\rangle=\left|\mu_{\mathrm{kn}}\right| / \mathrm{e}=\int_{\Omega} \psi_{\mathrm{n}} y \psi_{\mathrm{k}} \mathrm{d} \Omega$

with $y=$ Cartesian displacement $\Omega=$ Integration space around atom where wave-functions are non-zero $\psi_{\mathrm{n}}$, $\psi_{\mathrm{k}}=$ Wave functions for atomic states $\mathrm{n}$ and $\mathrm{k}$. Using Eq. (2) in our relations facilitates using the dimensionless ratio $e^{2} /(\hbar \mathrm{c})=1 / 137$ in the rest of the expressions. The spontaneous $v \underline{v}$-pair emission of (1) involves a second-order exchange of three quanta like in the Raman Effect, and is weaker compared to a first-order two-quantum interaction in the spontaneous emission of a single photon with probability [14]:

$$
\begin{aligned}
{ }^{\text {spon }} \mathrm{k}_{\mathrm{kn}}^{(\gamma)} & =(8 \pi / 3)\left\{e^{2} /(\hbar c)\right\}\left\langle y_{\mathrm{kn}}\right\rangle^{2} E_{\mathrm{kn}}^{3} /\left(\hbar^{3} \mathrm{c}^{2}\right) \\
& =2.379 \times 10^{7}\left\langle y_{\mathrm{kn}}\right\rangle^{2} E_{\mathrm{kn}}^{3}, s^{-1}
\end{aligned}
$$

Their ratio is:

${ }^{\left({ }^{(v)}\right)} \mathrm{k}_{\mathrm{kn}} /{ }^{(\gamma)} \mathrm{k}_{\mathrm{kn}}=0.940 \times 10^{-7}\left\langle y_{\mathrm{kn}}\right\rangle^{2} E_{\mathrm{kn}}^{2}$

with $E_{\mathrm{kn}}$ in eV energy units, and $\left\langle y_{\mathrm{kn}}\right\rangle$ in $\AA$. For typical values of $\left\langle y_{\mathrm{kn}}\right\rangle \approx 1 \AA$ and $E_{\mathrm{kn}} \approx 1 \mathrm{eV}$, it is clear that 
such a small $v \underline{v}$-pair emission probability is difficult to detect. This is as expected, since, otherwise discrepancies in the energy conservation laws of photon emission would have been noticed long ago.

\subsection{Stimulated emission of a neutrino pair}

Next, we consider stimulated emissions of a $\underline{v} \underline{\underline{p}}$ pair due to the interaction of a resonant photon with an excited atom or molecule. Using the semi-classical theory of radiation, one calculates that the probability of such a photon-stimulated $v \underline{v}$-pair emission for an electronic transition between energy levels $E_{k}$ and $E_{n}$ (with $E_{k}>E_{n}$ ) can be expressed by:

$$
\begin{aligned}
{ }^{(\gamma \rightarrow \underline{v})} \mathrm{k}_{\mathrm{kn}} & =\left[2^{6} \pi^{3} e^{4} /\left(3 \hbar^{5} c^{4}\right)\right]\left\langle y_{\mathrm{kn}}\right\rangle^{4} E_{\mathrm{kn}}^{2} \mathrm{I}_{\mathrm{L}}\left(\omega_{\gamma}=\omega_{\mathrm{kn}}\right) / \Delta \omega_{\mathrm{kn}} \\
& =0.2853\left\langle y_{\mathrm{kn}}\right\rangle^{4} E_{\mathrm{kn}}^{2} \mathrm{I}_{\mathrm{L}}\left(\omega_{\gamma}=\omega_{\mathrm{kn}}\right) / \Delta \omega_{\mathrm{kn}}, s^{-1}
\end{aligned}
$$

Again in the second expression, $\left\langle y_{\mathrm{kn}}\right\rangle$, is in Angstroms, $E_{\mathrm{kn}}=E_{\mathrm{k}}-E_{\mathrm{n}}$ is in $\mathrm{eV}$, while $\mathrm{I}_{\mathrm{L}}$ is laser power intensity in Watts $\mathrm{cm}^{-2}$, and $\Delta \omega_{\mathrm{kn}}$, is in $\mathrm{cm}^{-1}$. For a neodymium laser for example with $\left\langle y_{\mathrm{kn}}\right\rangle \approx 1 \AA, E_{\mathrm{kn}} \approx 1 \mathrm{eV}, \mathrm{I}_{\mathrm{L}}=1 \mathrm{~W} \mathrm{~cm}^{-2}$, and $\Delta \omega_{\mathrm{kn}} \approx 1 \mathrm{~cm}^{-1}$, one thus, finds that the probability of producing $v \underline{v}$ pairs is $0.2853 \mathrm{~s}^{-1}$ per lasing atom. This compares with the photon-stimulated photon emission rate per lasing atom of $3 \times 10^{6} \mathrm{~s}^{-1}$ according to the following relation [14]:

$$
\begin{aligned}
{ }^{(\gamma \rightarrow \gamma)} \mathrm{k}_{\mathrm{kn}} & =\left[\left(4 \pi^{2} / 3 \hbar\right)\left(e^{2} / \hbar c\right)\right]\left\langle y_{\mathrm{kn}}\right\rangle^{2}\left[I_{\mathrm{L}}\left(\omega_{\gamma}=\omega_{\mathrm{kn}}\right) / \Delta \omega_{\mathrm{kn}}\right] \\
& =3.035 \times 10^{6}\left\langle y_{\mathrm{kn}}\right\rangle^{2}\left[I_{\mathrm{L}}\left(\omega_{\gamma}=\omega_{\mathrm{kn}}\right) / \Delta \omega_{\mathrm{kn}}\right], s^{-1}
\end{aligned}
$$

Thus, the ratio of the two emission probabilities is:

${ }^{(\gamma \rightarrow v \underline{v})} \mathrm{k}_{\mathrm{kn}} /{ }^{(\gamma \rightarrow \gamma)} \mathrm{k}_{\mathrm{kn}}=0.940 \times 10^{-7}\left\langle y_{\mathrm{kn}}\right\rangle^{2} E_{\mathrm{kn}}^{2}$,

with $\left\langle y_{\mathrm{kn}}\right\rangle$ in $\AA$ units, $E_{\mathrm{kn}}$ in $\mathrm{eV}, \mathrm{I}_{\mathrm{L}}$ in Watts $\mathrm{cm}^{-2}$, and $\Delta \omega_{\mathrm{kn}}$ in $\mathrm{cm}^{-1}$ again in the last part of (6) and in (7). As expected, this ratio is the same as for spontaneous emissions.

In $v \underline{v}$-pair emissions, the resonant stimulating photon has energy $\hbar \omega_{\gamma} \approx E_{\mathrm{k}}-E_{\mathrm{n}}=E_{\mathrm{kn}}$ and it is postulated that this photon leaves the encounter unchanged with energy $\hbar \omega_{\gamma}$. Assuming $\epsilon_{v}=\hbar \omega_{v}$, we then have $\hbar \omega_{v}=\hbar \omega_{\underline{v}}=1 / 2 E_{\mathrm{kn}}$ or $\omega_{v}=1 / 2 E_{\mathrm{kn}} / \hbar=1 / 2 \omega_{\gamma}$, each (anti)neutrino taking half of the available atomic transition (exciton) energy of $E_{\mathrm{kn}}$. On the other hand if $\epsilon_{v}=1 / 2 \hbar \omega_{v}$, the $\underline{v}$-pair emitted in the stimulated emission would have energies $1 / 2 \hbar \omega_{v}=1 / 2 \hbar \omega_{\gamma}=1 / 2 E_{\mathrm{kn}}$, and the neutrino undulation frequency is the same as that of the laser photons, i.e. $\omega_{v}=\omega_{\gamma}=E_{\mathrm{kn}} / \hbar$. In both cases, the transition involves a three-quantum interaction with conversion of an exciton into a neutrino + antineutrino pair, stimulated by a resonant incident photon which remains unaffected. But in the first case, the detection laser (DL) must operate at a different resonant frequency $\omega_{\mathrm{DL}}=\omega_{v}=1 / 2 E_{\mathrm{kn}} /$ $\hbar=1 / 2 \omega_{\mathrm{SL}}$ instead of $\omega_{\mathrm{DL}}=\omega_{\mathrm{SL}}=E_{\mathrm{kn}} / \hbar$.

It may be possible that the incoming photon with energy $\hbar \omega_{\gamma}$ together with the exciton with energy $E_{\mathrm{kn}}$ are converted into a neutrino-antineutrino pair. Then if one assumes energies $\epsilon_{v}=\hbar \omega_{v}=\hbar \omega_{\underline{v}}$, one finds that $\omega_{v}=$ $\omega_{\underline{v}}=\omega_{\gamma}=E_{\mathrm{kn}} / \hbar$ must hold. [If $\epsilon_{\mathrm{v}}=1 / 2 \hbar \omega_{v}=1 / 2 \hbar \omega_{\underline{v}}$ one finds $\left.\omega_{v}=2 \omega_{\gamma}=2 E_{\mathrm{kn}} / \hbar\right]$. However, this is a four-quantum exchange (photon + exciton) $\rightarrow$ (neutrino + antineutrino), which has a lower probability than a threequantum (exciton) $\rightarrow$ (neutrino + antineutrino) exchange interaction by a factor $\sim 10^{-2}$ or less.

While $\omega_{v}=1 / 2 \omega_{\gamma}=1 / 2 \omega_{\mathrm{SL}}=1 / 2 E_{\mathrm{kn}} / \hbar$ appears most likely, it is uncertain which mechanism (a 3- or 4-quantum exchange) might produce a $\underline{v} \underline{v}$ pair emission. If $\omega_{v}=\omega_{\gamma}$, the detection laser (DL) can advantageously use the same laser medium as the SL, with $\omega_{\mathrm{DL}}=\omega_{\mathrm{SL}}=\omega_{v}=\omega_{\gamma}$ (as was postulated in the first tests at Korad in 1971-see Sect. 1). Otherwise the laser frequency of the DL unit must equal $\omega_{\mathrm{DL}}=\omega_{v}=1 / 2 \omega_{\mathrm{SL}}=1 / 2 \omega_{\gamma}$. For a neodymium laser one finds from (7) that ${ }^{(\gamma \rightarrow v \underline{v})} \mathrm{k}_{\mathrm{kn}} /(\gamma \rightarrow \gamma) \mathrm{k}_{\mathrm{kn}} \approx 10^{-7}$. Thus, in a neodymium SL, one of every $10^{7}$ stimulated de-excitations should yield a $v \underline{v}$ pair instead of a photon. Though weak, these beams of neutrinos and antineutrinos are emitted monochromatically and coherently, traveling along the same laser axis and exiting at both ends from a cylindrical lasing medium. For a pulsed $1 \mathrm{MW}$ neodymium laser with an internal peak laser photon flux of $6 \times 10^{24}$ photons $\mathrm{cm}^{-2} \mathrm{~s}^{-1}$, one generates pulsed beams of $3 \times 10^{17}$ neutrinos $\mathrm{cm}^{-2} \mathrm{~s}^{-1}$ coming out of each end of the laser tube or rod, composed of a 50-50 mixture of neutrinos and antineutrinos.

\subsection{Stimulated de-excitations by single neutrinos} or antineutrinos

The probability of stimulated de-excitations and photon emissions due to interactions by single neutrinos or antineutrinos is:

$$
\begin{aligned}
\mathrm{k}_{\mathrm{kn}}^{(v \rightarrow \gamma)} & \left.=\left[\left(4 \pi^{2} / 3 \hbar\right)\left(\mathrm{e}^{2} / \hbar c\right)\right] y_{\mathrm{kn}}^{2} E_{\mathrm{kn}}\left[\varphi_{v}\left(\omega_{v}=\omega_{\mathrm{kn}}\right) / \Delta \omega_{\mathrm{kn}}\right)\right] \\
& \left.=4.862 \times 10^{-13} y_{\mathrm{kn}}^{2} E_{\mathrm{kn}}\left[\varphi_{v}\left(\omega_{v}=\omega_{\mathrm{kn}}\right) / \Delta \omega_{\mathrm{kn}}\right), \mathrm{s}^{-1}\right]
\end{aligned}
$$

where the neutrino flux $\varphi_{v}$ is in neutrinos $\mathrm{cm}^{-2} \mathrm{~s}^{-1}, y_{\mathrm{kn}}$ is in $\AA$ units, $E_{\mathrm{kn}}=E_{\mathrm{k}}-E_{\mathrm{n}}$ is in $\mathrm{eV}$, and the resonance spread $\Delta \omega_{\mathrm{kn}}$ is in $\mathrm{cm}^{-1}$. The main assumption here is that neutrinos do carry undulating electromagnetic energy just like photons except they possess spin $1 / 2$ instead of 0 or 1 for photons. Like photons however, they can induce the deexcitation of resonant lasable levels without being absorbed when $\omega_{v}=E_{\mathrm{kn}} / \hbar=\omega_{\mathrm{L}}$. That is, for neutrinos undulating at frequency $\omega_{v}$ that matches a laser-resonant 
transition frequency $E_{\mathrm{kn}} / \hbar=\omega_{\mathrm{L}}$ in a medium with a lasable $\mathrm{n} \rightarrow \mathrm{k}$ population inversion, the probability of stimulating a de-excitation of the upper lasing level $E_{\mathrm{k}}$ with emission of a photon with frequency $\omega_{\gamma}=E_{\mathrm{kn}} / \hbar=\omega_{v}$, should be the same as it is for resonant photons with $\omega_{\gamma}=E_{\mathrm{kn}} / \hbar=\omega_{\mathrm{L}}$ that cause lasing. Then the ratio between the probability of a photon-stimulated de-excitation with laser photon emission and that by a neutrino- or antineutrinostimulated one, depends only on the relative flux levels:

${ }^{\operatorname{stim}} \mathrm{k}_{\mathrm{kn}}^{(v \rightarrow \gamma)} /{ }^{\mathrm{stim}} \mathrm{k}_{\mathrm{kn}}^{(\gamma \rightarrow \gamma)}=\varphi_{v}\left(\omega_{v}=\omega_{\mathrm{kn}}\right) / \varphi_{\mathrm{L}}\left(\omega_{\mathrm{L}}=\omega_{\mathrm{kn}}\right)$

In summary, a pulsed neodymium SL laser with $y_{\mathrm{kn}} \approx 1 \AA, E_{\mathrm{kn}} \approx 1 \mathrm{eV}, \Delta \omega_{\mathrm{kn}}=1 \mathrm{~cm}^{-1}$ at $1 \mathrm{MW}$ peak, generating a peak coherent neutrino beam of $6 \times 10^{17}$ neutrinos $\mathrm{cm}^{-2} \mathrm{~s}^{-1}$, could stimulate as many as $3 \times 10^{17}$ extra de-excitations per second per $\mathrm{cm}^{2}$ in an aligned $\mathrm{CW}$ milli-watt DL laser which normally experiences $2.5 \times 10^{15}$ de-excitations $\mathrm{cm}^{-2} \mathrm{~s}^{-1}$ internally due to photonstimulated emissions. Such a neutrino pulse would perturb lasing action dynamics in the low-power DL laser which can be observed via the laser output from this second unit. The only question is if the undulation frequency $\omega_{v}$ of neutrinos generated in the first neodymium laser should equal the laser frequency $\omega_{\mathrm{L}}$ of a second (neodymium) laser, or if it should be half that value as discussed. In the latter (most likely) case, one must use a different (holmium) laser for the detection laser unit.

\section{Proposed experiments}

To verify our predictions about laser-generated neutrino beams, the following tests are planned:

\subsection{Experimental verification with neodymium- and holmium-doped solid lasers}

A pulsed neodymium laser with a peak pulse power of $\sim 1 \mathrm{MW}$ or higher can be considered for a possible SL as illustrated in Fig. 2. Such a laser should produce a strong collimated pulsed beam of monochromatic neutrino pairs. Assuming that the internal peak power of the neodymium laser is $\sim 1 \mathrm{MW} / \mathrm{cm}^{2}$ or an internal peak flux of $6 \times 10^{24}$ photons $/ \mathrm{cm}^{2}$ per second at $\lambda_{\mathrm{L}} \approx 1.06 \mu \mathrm{m}$, it should generate and emit a neutrino beam of $3 \times 10^{17}$ neutrinos $\mathrm{cm}^{-2}$ per second out of each end, according to Eq. (7). This coherent neutrino beam emerging from both ends of a cylindrical neodymium laser rod will propagate along the same axis as the photon laser beam.

To detect the emitted neutrino beam, a second lowpower CW detection laser (DL) will be placed in the path of the photon beam from the neodymium SL. The DL will be placed some distance away (e.g. $50 \mathrm{~cm}$ ) from the neodymium SL with its axis optically aligned with the SL laser output beam (Fig. 2). In the alignment procedure, the SL photon output beam should be highly attenuated before it enters the low-power DL laser to avoid damaging it. Assuming that $\epsilon_{v}=\hbar \omega_{v}=1 / 2 \hbar \omega_{\mathrm{SL}}$, a suitable DL unit would be a $\mathrm{CW}$ holmium-doped $\left(\mathrm{Ho}^{3+}\right)$ erbium-oxide laser with $\lambda_{\mathrm{L}}=2.123 \mu \mathrm{m}$, i.e. at half the laser frequency of the neodymium SL with wavelength $\lambda_{\mathrm{L}}=1.0615 \mu \mathrm{m}$. To avoid missing the discovery that neutrinos might carry energy quanta $\epsilon_{v}=1 / 2 \hbar \omega_{v}=1 / 2 \hbar \omega_{\text {SL }}$ as discussed in Sect. 2.2, possible detection of the SL neutrino beam should also be checked with a low-power CW neodymium DL laser.

After optical alignment of the SL and DL lasers, a lightblocking material (e.g. a brick) is placed in the path of the external SL laser beam, so that only neutrinos can pass through it to enter the DL laser as shown in Fig 2. A lowpower holmium-based continuous DL laser might typically emit about $1 \mathrm{~mW} / \mathrm{cm}^{2}=1.2 \times 10^{16}$ photons $/ \mathrm{cm}^{2}$ per second near threshold with $\lambda_{\mathrm{L}}=2.12 \mu \mathrm{m}$. With $95 \%$ reflective end-mirrors, the intra-cavity DL flux level would then be about $1.2 \times 10^{17}$ photons $/ \mathrm{cm}^{2}$ per second. Then, when a pulsed SL neutrino beam enters the aligned DL with a flux of $3 \times 10^{17} \mathrm{~cm}^{-2} \mathrm{~s}^{-1}$ of resonant neutrinos, it can briefly enhance and depress (after excessive upperlevel depletion) the stimulated emission rate in the DL. This can be readily observed as a pulsed signal in the DL laser output. The microscopic cross-section for photonstimulated emissions as well as for neutrino-stimulated de-excitations is about $10^{-18} \mathrm{~cm}^{2}$. Since, the density of lasable excited states is $\sim 10^{16}$ holmium ions per $\mathrm{cm}^{3}$ for a near-threshold Ho laser, the macroscopic cross-section will be $\Sigma_{\mathrm{vstim}} \approx 10^{-2} \mathrm{~cm}^{-1}$ or the attenuation length $l_{\mathrm{mfp}} \approx 10^{2} \mathrm{~cm}=1 \mathrm{~m}$. With these parameters, a $10-\mathrm{cm}-$ long holmium-doped erbium-oxide DL rod aligned with the SL, would experience $\sim 3 \times 10^{16}$ additional emissions per $\mathrm{cm}^{2}$ per second during passage of the neutrino pulse of $3 \times 10^{17}$ neutrinos $\mathrm{cm}^{-2} \mathrm{~s}^{-1}$ from the SL. The internal DL laser photon flux of $\sim 10^{17}$ photons $\mathrm{cm}^{-2} \mathrm{~s}^{-1}$ will then be perturbed briefly during passage of the SL pulsed neutrinos, which can be recorded by the DL laser output as shown in Fig. 2. That is, during passage of the neutrinostimulated de-excitation pulse, the DL output should briefly go up and then dip down before returning to its original level.

To insure that electrical interference from the pulsed SL does not produce a false signal on the power meter of the DL, it is important that complete electromagnetic isolation between the electronic circuits of the pulse generator and the detector's oscilloscope be maintained. Separate batteries might be used to empower these circuits. Clearly before any neutrino experiments are conducted, the aligned SL and DL systems should be tested to assure there is no mutual electronic or optical interference. 
3.2 Experimental verification with $\mathrm{CO}$ and $\mathrm{CO}_{2}$ gas lasers

Another suitable pair of SL/DL lasers is a $\mathrm{CO}$ laser with wavelength $\lambda_{\mathrm{L}} \sim 5.3 \mu \mathrm{m}$ as the SL unit, and a $\mathrm{CO}_{2}$ laser with $\lambda_{\mathrm{L}} \sim 10.6 \mu \mathrm{m}$ as the DL unit. The $\mathrm{CO}_{2}$ laser has a large number of rovibrational lines of which some have frequencies that coincide with half the frequency of some of the CO laser's rovibrational lines. For example, the CO laser line at $\lambda_{\mathrm{L}}=5.44239 \mu \mathrm{m}$ produced by CO's rovibrational transition $(\mathrm{v}=11, \mathrm{~J}=13) \rightarrow(\mathrm{v}=10, \mathrm{~J}=12)$ is nearly coincident with half of the wavelength $\lambda_{\mathrm{L}}=10.88473 \mu \mathrm{m}$ produced by $\mathrm{CO}_{2}$ 's lasable rovibrational transition $\left(\mathrm{v}_{3}=1, \mathrm{~J}=46\right) \rightarrow\left(\mathrm{v}_{1}=1, \mathrm{~J}=45\right)$. Here $\mathrm{J}$ is the rotational quantum number of a $\mathrm{CO}$ or $\mathrm{CO}_{2}$ molecule and $\mathrm{v}$ is its vibrational level. A drawback of these mid-infrared lasers is that $E_{\mathrm{kn}}=0.1-0.2 \mathrm{eV}$ compared to $E_{\mathrm{kn}} \sim 1 \mathrm{eV}$ for a near-infrared neodymium laser. According to Eq. (4), the neutrino flux emitted by a pulsed CO SL is then lower by a factor $\sim 10^{-9}$ instead of $\sim 10^{-7}$ relative to the intra-cavity laser photon flux. Peak intracavity power levels of $100 \mathrm{~kW} / \mathrm{cm}^{2}$ or $2.86 \times 10^{24}$ photons $\mathrm{cm}^{-2} \mathrm{~s}^{-1}$ generated by a pulsed Q-switched CO laser would, thus, produce $2.86 \times 10^{15}$ neutrinos $\mathrm{cm}^{-2} \mathrm{~s}^{-1}$. A continuous $\mathrm{CO}_{2}$ laser operating at the $10.88473 \mu \mathrm{m}$ line with intracavity flux of $10^{15}$ photons $\mathrm{cm}^{-2} \mathrm{~s}^{-1}$ or $0.07 \mathrm{~mW} / \mathrm{cm}^{2}$ could detect such fly-through neutrinos.

Again, to investigate the possibility that neutrinos carry energy quanta $\epsilon_{\mathrm{v}}=1 / 2 \hbar \omega_{v}=1 / 2 \hbar \omega_{\mathrm{SL}}$ instead of $\epsilon_{v}=\hbar \omega_{v}=1 / 2 \hbar \omega_{\mathrm{SL}}$ as discussed in Sect. 2.2, detection of the SL neutrino beam should be checked with a low-power continuously running $\mathrm{CO}$ detection laser as well. As before, a laser beam blocking object like a thick solid brick should be placed between the aligned $\mathrm{CO}$ laser source and the $\mathrm{CO}_{2}$ (or $\mathrm{CO}$ ) detection laser to assure that only non-absorbable neutrinos enter the DL. Following axial alignment of the DL and SL lasers (placed about $50 \mathrm{~cm}$ apart), the DL unit should be totally shielded optically from any infrared laser photons emitted by the SL unit. Not a single SL laser photon should be sensed by the photo-detector that monitors the laser power of the DL laser. As before, great care must also be taken that no electrical noise from the highpower pulsed SL is picked up by the DL photo-detector. In earlier exploratory tests, laser photons from the high-power pulsed SL laser had scattered off the beam blocker placed between the SL and DL units. After re-scattering from nearby walls and other objects, some of these photons were still able to enter the DL's photo-monitor, giving a false neutrino signal. This possibility must be eliminated to assure that the weak fly-through neutrino beam signals in the DL are not overwhelmed by weak signals from stray SL photons.
3.3 Experimental re-verification of reactor-produced low-energy neutrino interactions with lasers

The unexplained quenching of lasing due to high-power TRIGA research reactor pulses observed at General Atomics in 1964 [13] as described at the end of Sect. 1, should be re-investigated with the view that low-energy neutrinos produced during fission may cause such disruptions. Recently, truck-mounted high-energy $(\mathrm{MeV})$ neutrino detection chambers have been deployed near nuclear power reactors to monitor U-235/Pu-239 loadings. A similar mobile $\mathrm{MeV}$ neutrino detector might be used to follow the pulsed neutrino outputs of a TRIGA reactor for our present research to verify and explore the laser-quenching by lowenergy (sub-keV) reactor neutrinos. The pulsed fluxes of low-energy neutrinos are proportional to and can be derived from the high-energy $\mathrm{MeV}$ neutrino emission data.

Like Compton [13] did, a gamma- and neutron-shielded $\mathrm{CW}$-operated $\mathrm{Nd}$ or $\mathrm{CO}$ laser together with output power meter, should be placed in a light-tight box next to the TRIGA reactor core. The laser outputs should then be monitored as the TRIGA is pulsed to multi-megawatts of peak fission power. Before laser tests are conducted, the gamma and neutron flux levels inside the shielded lighttight box during a reactor pulse must be measured without any lasers present. This is to insure that the lead-shielding against gammas and boron-shielding against neutrons are adequate to avoid color-center formations in neodymium rods or adverse excited molecular populations in the $\mathrm{CO}$ laser gas that halts the lasing action. The lasers should then be subjected to the same residual pure gamma and pure neutron irradiation levels from independent non-reactor sources to insure that lasing is not interrupted by residual gammas and neutrons leaking through shielding around the box. If such interruptions do not occur, then with an operating laser in the box and with the TRIGA pulsing, one has reasonable assurance that any observed lasing stoppage must be caused by fly-through neutrinos and not by leaked reactor gammas or neutrons.

\section{Conclusions}

Based on the revised analysis of an earlier neutrino physics study [1], and some puzzling 1964 experiments of the interaction of a pulsed TRIGA reactor with lasers at General Atomics [13], an experimental demonstration of the generation and detection of low-energy coherent neutrino beams with the assistance of lasers is indicated. It would considerably expand our knowledge of these elusive quanta and of physics in general. A positive outcome of such tests would conclusively settle the question whether neutrinos 
are of electromagnetic origin and can be active in the atomic as well as nuclear domain. If the proposed detection of neutrinos via stimulated emission is successful, one can also ascertain whether their energy quanta are related to their undulation frequencies by $\epsilon_{v}=\hbar \omega_{v}$ or $\epsilon_{v}=1 / 2 \hbar \omega_{v}$.

Because they are not absorbable by molecular or atomic matter (except by nuclei if $\epsilon_{v}>1 \mathrm{MeV}$ ) and can travel unimpeded through the earth, seas, and mountains, neutrino beams could be used as information carriers using techniques similar to those employed in today's photonic broadcasts of information [10]. Non-absorbable beams of bunched coherent neutrinos may still experience diffractions and refractions and could be used to map out ore bodies and other interior features of the earth, mountains, or oceans. Just like the laser was initially looking for an application after its debut in 1960, so the coherent neutrino beams are expected to find many other applications besides fly-through transmissions of information or geophysical probing.

Open Access This article is distributed under the terms of the Creative Commons Attribution License which permits any use, distribution, and reproduction in any medium, provided the original author(s) and the source are credited.

\section{References}

1. J.W. Eerkens, Neutrino communications proof-of-principle experiment. Document XN- PR-67-0017, Unsolicited Proposal to
ARPA by Xion Corporation (Founders/principals: G.T. Roth, J.W. Eerkens, E.G. Erickson, P.E. Sweitzer, G. De Vito, D. Hess), Sep 18, (1967)

2. M.L. de Broglie, Une Nouvelle Conception de la Lumiere, et "Sur la Nouvelle Theorie du Photon. Comptes Rendus Acad. Sci. Paris 195, 862; 197, 536; 197, 1377; 198, I35; 199, 445; 199, 1165 (1932-1934)

3. P. Jordan, Neutrinotheorie des Lichtes. Z Physik 93, 464; 98, 759; 99, 109; 102, 243; 105, 114; 105, 229 (1932-1937)

4. R. de Laer Kronig, Zur Neutrino Theorie des Lichtes. Parts I, II, III, Physica 2, 491, 854, 968 (1935)

5. M. Born, N.S. Nagendra Nath, The neutrino theory of light. Proc. Ind. Acad. Sci. 3, 318; 3, 448; 3, 611 (1936)

6. W.A. Perkins, Neutrino theory of photons. Phys. Rev. 137(5B), B1291 (1965)

7. B. Ferretti, I. Venturi, On the possibility of a neutrino theory of light. Nuovo Cimento XXXV(2), 644 (1964)

8. H.M.L. Pryce, On the neutrino theory of light. Proc. R. Soc. Lond. A-165, 247 (1938)

9. V.S. Berezinskii, Pryce's theorem and the neutrino theory of photons. Sov. Phys. JETP 24(5), 927 (1965)

10. J.W. Eerkens, Neutrino communication arrangement. US Patent 3,430.046, issued in 1980

11. W.A. Perkins, Statistics of a composite photon formed of two fermions. Phys. Rev. 5(6), 1375 (1972)

12. E.J. Sternglass, Electron-positron model for the charged mesons and pion resonances. Nuovo Cimento XXXV(1), 227 (1965)

13. D.M.J. Compton, Coherent light transmitter techniques. General Atomics, Technical Report AFAL-TR-64-318, prepared for Air Force Avionics Lab, AF Systems Command, WPAFB, Ohio, (Jan 11, 1965)

14. J.W. Eerkens, Model equations for photon emission rates and absorption cross-sections; Vol II Rocket radiation handbook. US Air Force FTD, No CW-01-01-74; CDC No AD-A007946, Dec 1973 (AF Contract F33657-72-C-0850) 